\title{
Acquisition and Data Logging Inertial Navigation System (INS) on Combat Submarine Using Arduino-SD Card
}

\author{
Varisa Rahmawati ${ }^{1}$, Achmad Affandi ${ }^{2}$, Imam Arifin ${ }^{1}$, and Muhammad Asrofi ${ }^{3}$
}

\begin{abstract}
In a combat submarine operating system, navigation device acts as a location information provider on inside or at sea level. Such information is position data provided by GPS (Global Positioning System) and data transmitted by IMU (Inertial Measurement Unit) sensor. Combining these two device is called INS (Inertial Navigation System). This inertial sensor interacts using serial communication data, however records are needed to collect, store and store incoming data in order to be processed easily. Data logging process is feasible as a recorder and data storage using SD Card. Enhancements, such as SD cards are needed because in general system is equipped with a small memory capacity. Arduino is a microcontroller that can assist in process because data obtained from sensor is processed using serial communication lines $(R X$ and $T X)$. Results obtained from calculation and logging data are calculation Roll, Pitch, Heading, Latitude, Longitude, Microseconds, Gyroscope on $X$ axis, $Y, Z$, Accelerometer on $X$ axis, $Y, Z$, Magnetometer on $X, Y, Z$, Velocity North, East, Down. This proves that system acceleration and data logging using Arduino and SD Card can assist in obtaining information and $\mathrm{KTBA}$ situations.
\end{abstract}

Keywords-

\section{INTRODUCTION}

Combat submarine is a type of submarine that functions as a defense of Indonesia's conservation areas. Buoyant force on this combat submarine can be regulated, causing pollution and flooding needs. However, light can't penetrate deeper into oceans, consequently combat submarine must be controlled through air with blind views. Combat submarine navigation tools are an important tool in determining position on surface of earth and at sea. Navigation system on combat submarine are needed to get latest position data and also vessel data. In research number one utilizing from INS and DVL (Doppler Velocity Log) to request information about location of submarine. Through a system between INS and DVL, sensor reaches an accuracy as $0.08 \%$ of total distance and is more accurate than other sensors. Data communication combines two systems using RS 232 and RS 422 [1]. Literature number two, research on $\mathrm{pH}$ data designers and logging devices using a microcontroller. This

\footnotetext{
${ }^{1}$ Varisa Rahmawati and Imam Arifin are with Department of Automation Electrical Engineering, Institut Teknologi Sepuluh Nopember, Surabaya, 60111, Indonesia. E-mail: varisarahmawati@gmail.com.

${ }^{2}$ Achmad Affandi is with Department of Electrical Engineering, Institut Teknologi Sepuluh Nopember, Surabaya, 60111, Indonesia.

${ }^{3} \mathrm{M}$. Asrofi is with Staff of PT Bhimasena Research and Development.
}

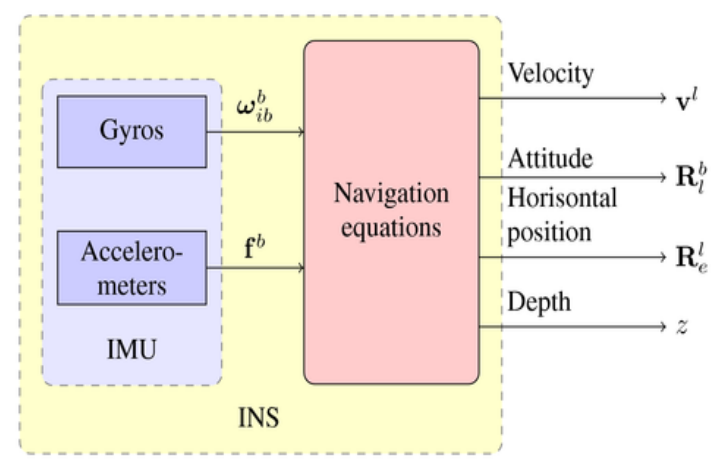

Figure 1. Inertial Navigation System.

includes making data and data logging to allow analysis and sharing of data. The device used is Renesas M16C / 62P microcontroller. In microcontroller there is an EEPROM (Electrically Erasable Programmable Read-Only Memory) as a medium for logging data or storing and finding ADC as an analog to digital conversion value. Data acquisition includes process of extracting, transforming and transporting data from system and external data to microcontroller for storage. This data can be recorded in real time or offline by downloading EEPROM memory [2]. The navigation device used in this research is INS, this device is a combination of GPS performance and IMU sensor [3]. To get data from sensor, acquisition system design is used as information retrieval. One device that can support this system, namely Arduino [4]. This device is a microcontroller that is capable of serial communication with sensor. Arduino has internal memory with limited storage capacity of $256 \mathrm{~KB}$ [5]. This prevents user from analyzing data from sensor. Sensor performance analysis is very necessary to know the response to the motion condition that occur on submarine. A media logging is used, which is a data logger, therefore data storage can be done. Thus, stored data can be analyzed offline [4]. This paper discusses the performance of INS and data acquisition and logging system found in section II. Next, section III discussed implementation of data acquisition and logging systems for combat submarine. Section IV is a testing and results process, while section $\mathrm{V}$ is the conclusion of the research.

\section{INERTIAL NAVIGATION SYSTEM}

The inertial navigation system can provide the position and speed on combat submarine. By processing signal from this 
device [6], inertial navigation can rely on initial position knowledge, speed, and attitude. Illustrated INS in Figure 1.

In INS, angular levels and specific strength measurements from IMU are processed to produce a state of position, speed and submarine attitude. There are three gyroscope axes, and a magnetometer and GPS receiver that is used as a monitoring tool for position and attitude [4]. GPS receiver gives the initial position before the dive boat is accurate without manual entry. Inertial sensor has 3 axes, namely $X$, $\mathrm{Y}$, and $\mathrm{Z}$ as a determinant of the direction around the measured angle and acceleration. Output from INS is a series of bytes, therefore data can not be translated directly by user.

\section{A. Data Acquisition}

Acquisition system consists of sensor, measurement hardware, and computer with programmable software. In actual terms, the acquisition is in form of an interface between environment analog to digital environment. In retrieving and processing data from INS, system design is needed to convert physical signal into electronic signal. Next, the signal is processed by computer into bit code and other numbers.

\section{B. Logging Data}

This process aims to archive data that has been taken for analysis or further processing offline. Data logging is the application of measurement and recording of data or physical or electrical parameter over a specified period of time [8]. Results of the acquisition or retrieval of data from INS, then stored in data logger, is SD Card. Data Logger is an electronic instrument that records measurements over time. On the other hand data logger have ability to receive more input channel, with better resolution and accuracy.

\section{IMPLEMENTATION OF ACQUISITION AND DATA LOGGING}

Whole system can be illustrated in Figure 2, where sensor used is Sublocus and system controller is Arduino. SD Card module is a data storage medium from Sublocus, where data can be displayed on a computer.

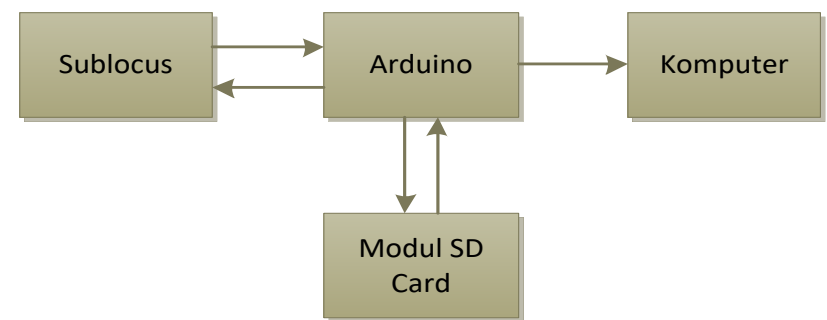

Figure 2. Block Diagram of Data Acquisition and Logging System.

Sublocus data can be sent in ANP Protocol format via RS 422 communication line to Arduino. This protocol helps in process of transmitting data from Sublocus to other devices. The results of data are taken and processed by Arduino microcontroller, then displayed on serial monitor on computer. In addition, data can be stored on SD Card modules all the time continuously.

\section{A. Data Acquisition}

Device configuration of different data processing is seen in Figure 3. Data from INS is processed using Arduino microcontroller based on ATmega 2560, where between INS and Arduino device there is RS 422 module.

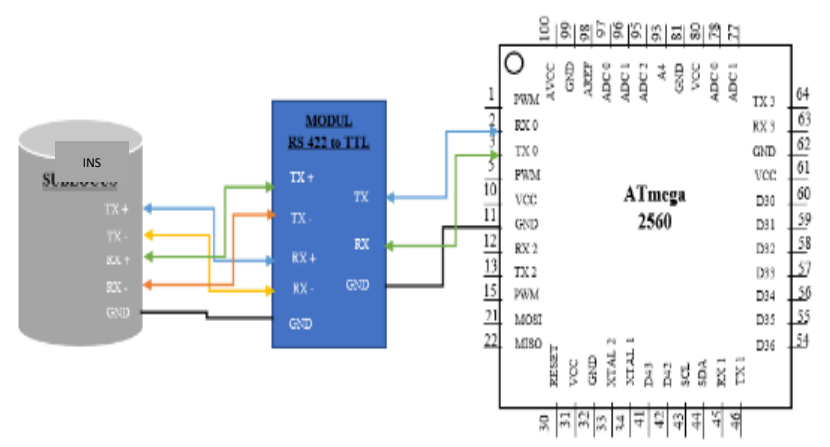

Figure 3. Configuration of Data Acquisition System.

This acquisition system use INS sensor which has 5 data path, while for transmission to Arduino require 3 data path, that is TX, RX, and GND. Therefore RS 422 module to TTL is used to facilitate input and output configuration between INS and Arduino. In communicating from this device, a program is needed to give commands to Arduino board. Acquisition system program flowchart can be seen in Figure 4.

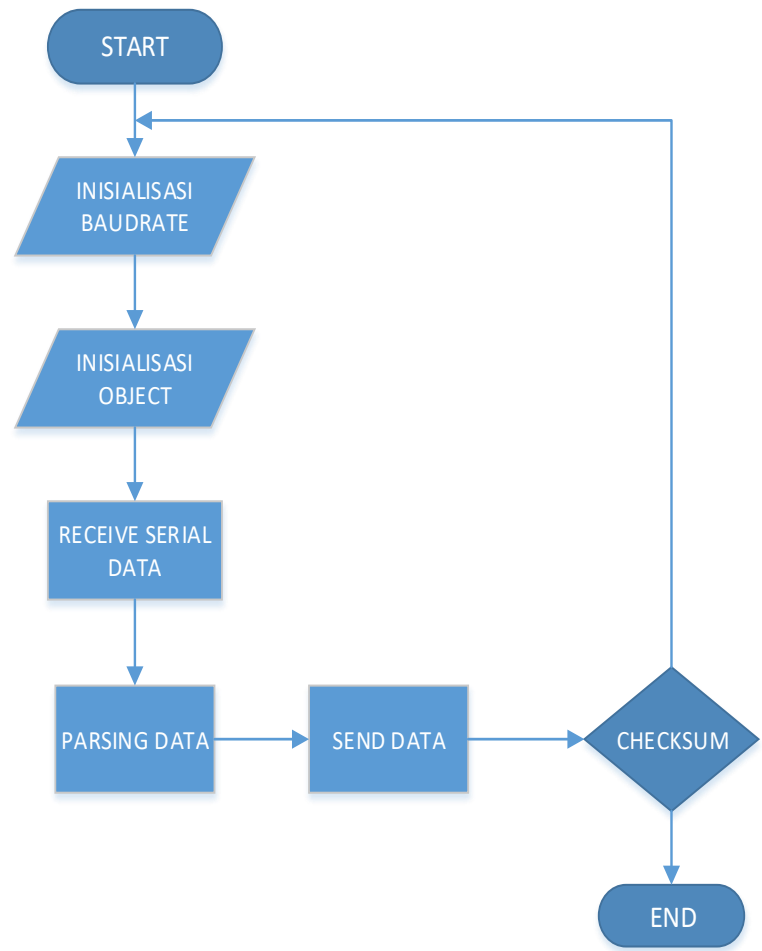

Figure 4. Flowchart of Data Acquisition.

Purpose to process incoming data packets by separating data packets into descriptions of data that is processed to the next stage. When stage has been done, next step, namely data transmission. 


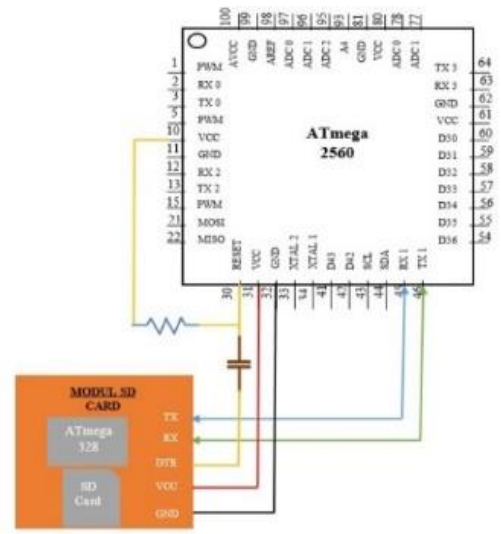

Figure 5. Configuration of Data Logging System.
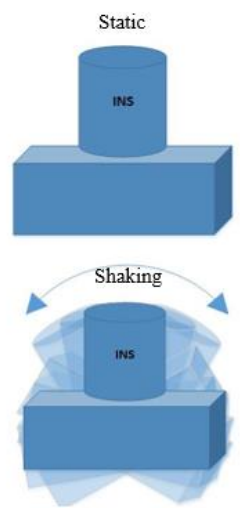

Figure 6. Illustration Testing Four Conditions.

TABLE 1.

DATA ACQUiSITION AND LOGGING RESUlTS DATA

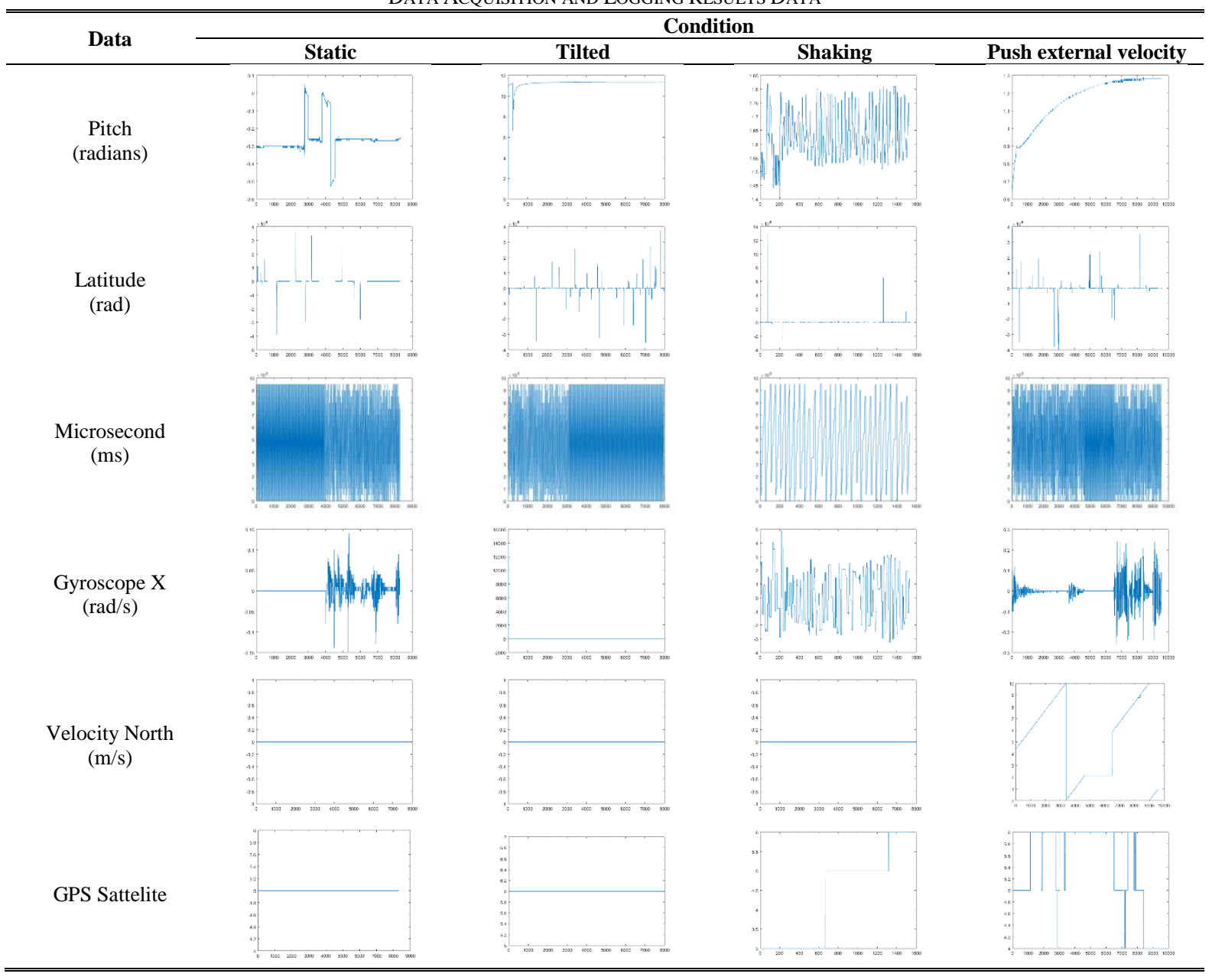

\section{B. Data Logging}

SD Card module is connected to Arduino based ATmega 2560. It is a data logging design using SD Card Module media, which in module consists of ATmega 328 and SD Card. In Figure 5 shows configuration of data logging system. This data logger system is built from Arduino module as a system controller and use SD Card as a data storage medium. When data recording process is carried out, data from obtained Subocus is sent to RX TX port, and received by SD Card module.

\section{TESTING AND RESULT}

Testing data acquisition and recording is done by providing four different INS conditions or circumstances. This 
International Conference on Engineering, Advance Science and Industrial Application (ICETESIA) 2018

September 6-7 2018, Institut Teknologi Sepuluh Nopember, Surabaya, Indonesia

condition when Sublocus is in a static, tilted, when state of interference or shocks, and push external velocity. Illustrations of four conditions can be seen in Figure 6.

Data taken are Roll, Pitch, Heading, Latitude, Longitude, Microseconds, Gyroscope X, Gyroscope, Gyroscope Z, Acceleration $\mathrm{X}$, Acceleration $\mathrm{Y}$, Acceleration Z, Magnetometer X, Y Magnetometer, Z Magnetometer, GPS Sattelite, Velocity North, East, Down. This test is done by observing response of 19 data that has been obtained in accordance with influence of given Sublocus conditions. Table 1 shows results of data acquisition and logging.

\section{CONCLUSION}

Data can be taken and stored using Data Acquisition and Logging system through Arduino Mega, RS 422, and SD Card Module. Data acquisition and logging systems can display data results in the form of Roll, Pitch, Heading, Latitude, Longitude, Microsecond, Gyroscope on X, Y, Z axis, accelerometer X, Y, Z axis, Magnetometer on X, Y, Z axis, GPS Sattelite Velocity North, East, Down.

\section{REFERENCE}

[1] D. Kuil, "The implementation of an underwater navigation system, applied to Ortega's submersibles," pp. 1-41.

[2] C. S. Misal and J. M. Conrad, "Designing a pH data acquisition and logging device using an inexpensive microcontroller," Conf. Proc. - IEEE Southeastcon, pp. 217-220, 2007.

[3] R. Panish and M. Taylor, "Achieving high navigation accuracy using inertial navigation systems in autonomous underwater vehicles," Ocean. 2011 IEEE - Spain, pp. 1-7, 2011.

[4] S. P. Ambildhok and N. B. Hulle, "Development of Inertial Navigation System based on Accelerometer and Gyroscope," vol. 7, no. 5, pp. 12149-12151, 2017.

[5] M. Windows et al., "Aurduino mega 2560," Uma ética para quantos?, vol. XXXIII, no. 2, pp. 81-87, 2014.

[6] N. Syahroni, W. P. Yuniar Riska, P. I. Metha, H. W. Suparno, H. Budiman, and C. J. Weon, "Data acquisition and processing of movement and position for AUVs with experiment results," ICITACEE 2015 - 2nd Int. Conf. Inf. Technol. Comput. Electr. Eng. Green Technol. Strength. Inf. Technol. Electr. Comput. Eng. Implementation, Proc., pp. 97-101, 2016. 